\title{
Isolation and expression analysis of the soybean GmPic gene
}

\author{
A.-H. Sha ${ }^{1,3 *}$, H. Wu ${ }^{2 *}$, X.-M. Fu², Q.-L. Zhang' ${ }^{2}$, Q.-L. Guo ${ }^{2}$, Y.-H. Chen ${ }^{2}$ \\ and X.-A. Zhou ${ }^{1}$ \\ ${ }^{1}$ Institute of Oil Crops Research, Chinese Academy of Agriculture Sciences, \\ Wuhan, Hubei, China \\ ${ }^{2}$ Hainan Key Laboratory for Sustainable Utilization of Tropical Bioresource, \\ Hainan University, Haikou, China \\ ${ }^{3}$ College of Agriculture, Yangtze University, Jingzhou, China \\ *These authors contributed equally to this study. \\ Corresponding authors: Y.-H. Chen / A.-H. Sha \\ E-mail: aihuasha9@163.com
}

Genet. Mol. Res. 13 (2): 4380-4391 (2014)

Received June 12, 2013

Accepted December 4, 2013

Published June 11, 2014

DOI http://dx.doi.org/10.4238/2014.June.11.2

\begin{abstract}
The differential screening method was used to isolate the soy photoperiodic response-related genes and to further elucidate the molecular mechanisms of the soybean photoperiodic response. The light-sensitive species Zhong Dou 24 was used to receive longtime sunshine, short-time sunshine, and natural sunshine treatment. The cDNA-amplified fragment length polymorphism technique was used to screen the differentially expressed cDNA fragments. The rapid amplification of cDNA end technique was used to isolate the gene. Semi-quantitative reverse transcription polymerase chain reaction analysis was used to analyze the gene expression patterns in different light cycles. The gene had a total length of $983 \mathrm{bp}$, contained a complete open reading frame that encoded 248 amino acids, and shared homology with the mitochondrial phosphate transporter protein. The expression pattern analysis results showed that this gene was expressed in the early stages of soybean growth and development. The shorttime sunshine inhibited its expression, whereas the long-time sunshine
\end{abstract}


enhanced its expression. The differential screening method was used to isolate the soybean mitochondrial phosphate transporter gene. The gene may be used as a negative regulatory factor that is involved in the photoperiodic response of soybean.

Key words: Soybean; Mitochondrial phosphate transporter protein; Photoperiod; cDNA-amplified fragment length polymorphism

\section{INTRODUCTION}

Soybean (Glycine max) is a typical plant that requires a short sunshine time. Its flowering time and maturity are important traits of agricultural production. Soybean controls seed production and parts of disease resistance; it also limits the mutual introduced promotion in different ecological environments and increases the cost of breeding. In order to expand the planting area and to improve the production, there is the need to broaden the photoperiod adaptability of soybean. According to research on several model plants such as Arabidopsis, snapdragon, and corn, four signaling pathways in the regulation of flowering time have been proposed: photoperiodic response pathway, vernalization response pathway, autonomous flowering pathway, and Gibberellins (GA) route (Garner and Allard, 1920; Pittendrigh and Minis, 1964; Hayama et al., 2002, 2003; Yanovsky and Kay, 2003; Hayama and Coupland, 2004; Hecht et al., 2005). These pathways ultimately acted on the major genes controlling flowering, such as constans (CO), leafy, apetala 1, flowering locus T (FT), and SOC1. These pathways will result in the transformation of the shoot apical meristem to the floral primordium. With respect to the methods that plants use to distinguish the day length and the mechanisms of flowering time regulation, it was generally believed that FT could induce the gene expression of the floral primordium differentiation, and $\mathrm{CO}$ could regulate FT expression. With the participation of many other genes, the phenomenon of the light cycle would be eventually formed (Kojima et al., 2002). Zhao et al. (2007) used the suppression subtractive hybridization technology to perform the differential screening of Dong Nong L13. Seventy-six dark-induced genes were obtained, and they were mainly cDNAs that were involved in developmental regulation, signal transduction, and apoptosis defense. They were a series of new genes involving conserved domain regulatory factors, receptor protein kinase, antioxidant enzymes, and some other unknown functions (Zhao et al., 2007). In addition, the soybean CO and FT homologs, GmCO and GmFT, the biological clock gene ZTL, and one member of the MADS-box gene family (GmNMH7) were cloned (Sha et al., 2006; Ke, 2006; Gao, 2008; Liu et al., 2008, 2009). However, the specific role of these genes in the soybean photoperiod response and its methods of floral primordial regulation require further investigation.

In this study, cDNA-amplified fragment length polymorphism (AFLP) technology was used to analyze the light-sensitive soybean variety Zhong Dou 24 to explore the gene expression under different light conditions. The mitochondrial phosphate transporter gene GmPic was cloned from soybean. The gene was expressed in the early stages of soybean growth and development. The short-time sunshine inhibited its expression and induced flowering, while the long-time sunshine enhanced its expression and thereby inhibited the flowering. It can be concluded that the gene may be used as a negative regulator, inhibiting flowering during the photoperiodic response. 


\section{MATERIAL AND METHODS}

\section{Experimental processing and material preparation}

The experimental material was Zhong Dou 24, which is a photoperiod-sensitive soybean. Seeds were sown on April 14, 2005 in the experimental field of oil crops research of Chinese Academy of Agricultural Sciences. After the first trifoliate grew, the plants received short-time sunshine $(8 \mathrm{~h}$ in light and $16 \mathrm{~h}$ in dark), long-time sunshine (16 h in light and $8 \mathrm{~h}$ in dark), and natural sunshine ( $13.5 \mathrm{~h}$ in light and $10.5 \mathrm{~h}$ in dark). The processing method was according to the study of Yang et al. (2001a,b). When the natural sunshine hours were longer than the planned sunshine hours, black PVC material was used to make sunshades for artificial shading. When the natural sunshine hours were shorter than the planned sunshine hours, a supplemental light was used. The light source was $250 \mathrm{~W}$ fluorescent high-pressure mercury lamp $\left(14 \mathrm{~m}^{2} / \mathrm{lamp}\right)$ that was placed $1.5 \mathrm{~m}$ above the ground. The light processing time was 20 days. The newly expanded leaves were obtained every 5 days after the light treatment until flowering. The obtained leaves were immediately frozen in liquid nitrogen and placed at $-80^{\circ} \mathrm{C}$ until further use. The date of flowering was recorded in the field, and the date of the first flower after treatment was taken as the flowering period.

\section{RNA extraction and cDNA-AFLP analysis}

The isolation of total RNA and double-stranded cDNA synthesis were performed with Trizol reagent (Roche, USA) and SMART cDNA synthesis kit (Clontech, USA) according to manufacturer instructions. cDNA-AFLP analysis was performed according to the methods described by Bachem et al. (1996), with minor modifications. Five hundred nanograms of double-stranded cDNA, $5 \mathrm{U}$ EcoRI, and $5 \mathrm{U}$ MseI (MBI, Lithuania) were put into the $25 \mu \mathrm{L}$ $1 \mathrm{X} \mathrm{Y}^{+} / \mathrm{TANGO}^{\mathrm{TM}}$ buffer at $37^{\circ} \mathrm{C}$ for $2.5 \mathrm{~h}$ of enzyme digestion. Twenty-five microliters of the ligation mixture including 5 pmol EcoRI connector (5'-CTCGTAGACTGCGTACC-3' plus 5'-AATTGGTACGCAGTCTAC-3'), 50 pmol MseI connector (5'-GACGATGAGTCCTGAG3' plus 5'-TACTCAGGACTCAT-3'), 1X ligation buffer, and 0.5 U T4 DNA ligase (MBI) was incubated at $37^{\circ} \mathrm{C}$ overnight. The ligation products were diluted 10 -fold with Trisethylenediaminetetraacetic acid (TE) and used as the pre-amplification template. The pre-amplification primers were MseI primer and EcoRI without selective bases. The preamplification products were diluted 10-fold with TE and used as the amplification template. The selected amplification volume was $20 \mu \mathrm{L}$; it included $4 \mu \mathrm{L}$ diluted pre-amplification products, $40 \mathrm{ng} \mathrm{M}_{(\mathrm{VN})}, 40 \mathrm{ng} \mathrm{E}_{(\mathrm{VN})}, 0.2 \mathrm{mM}$ dNTPs, 1X polymerase chain reaction (PCR) buffer, and $1 \mathrm{U}$ Taq enzyme (MBI). The core sequences of the $\mathrm{E}_{(\mathrm{VN})}$ and $\mathrm{M}_{(\mathrm{VN})}$ primers were the same as those in the MseI and the EcoRI primers of the pre-amplification, respectively. Two selective bases ( $\mathrm{V}$ and $\mathrm{N}$ representing bases $\mathrm{A} / \mathrm{G} / \mathrm{C}$ and $\mathrm{A} / \mathrm{T} / \mathrm{G} / \mathrm{C}$, respectively) were added at the 3 '-end. A total of 256 pairs of primer combinations were included. PCRs were performed according to those described by Vos et al. (1995) on the PTC-200 PCR apparatus. The selected amplified products were separated on $6 \%$ sequencing gels and stained with silver.

\section{Cloning and sequencing of differential fragments}

The differentially expressed fragments were obtained from the sequencing gel, and a clean 
knife was used to cut plastic blocks from the gel. The blocks were then placed in $1.5-\mathrm{mL}$ centrifuge tubes with $100 \mu \mathrm{L}$ sterilized ultrapure water. They were boiled for $15 \mathrm{~min}$ at $95^{\circ} \mathrm{C}$ and eluted. Two microliters of eluted cDNA was used as the template for PCR amplification. The amplification primers and PCR system were the same as the selected amplification. The PCR products were purified with a gel kit (V-Gene) and ligated with the pMD-18 vector (Takara). One hundred microliters of Escherichia coli $\mathrm{DH} 5 \mathrm{a}$ competent cells with $5 \mu \mathrm{L}$ of the ligation products were transformed and selected in 400 $\mu \mathrm{L}$ Luria broth (LB) culture medium at $37^{\circ} \mathrm{C}$ with oscillation ( 150 revolutions $/ \mathrm{min}$ ) for $1.5 \mathrm{~h}$. Two hundred microliters of bacterial suspension was applied to the LB culture plates containing ampicillin $(60 \mathrm{mg} / \mathrm{L}), \mathrm{X}$-gal, and isopropyl $\beta$-D-1-thiogalactopyranoside and incubated at $37^{\circ} \mathrm{C}$ for $16-20 \mathrm{~h}$; the white colonies were selected. The pMD-18 vector universal primers M13-47 and RV-M were used for PCR amplification of the picked colonies. The inserted fragment size of the positive clones was detected. The positive clones were sequenced using M13 forward or reverse primers by Shanghai Biological Engineering Technology Co., Ltd. The obtained sequence information was compared with the sequence in the nucleotide database using the basic local alignment search tool (BLAST).

\section{Reverse transcription (RT)-PCR analysis}

The total RNA was extracted from the soybean leaves with different light treatments and different developmental stages. After the DNase I (Promega) treatment, oligo dT (18) and M-MLV reverse transcriptase (Promega) were used for the synthesis of the first cDNA chain according to manufacturer instructions. The corresponding GmPic primers were used for RT-PCR analysis, and the soybean actin gene (gi18531) was used as a control. The amplification primers for GmPic were 5'-CAGACGCAGCCAAGTACAAT-3' and 5'-GTCCTGAGTAAATGCCGTTTG3'; the amplification primers for actin were 5'-GTATTGTATTGGACTCTGGTGATGG-3' and 5'-TTAGAAGCACTTCCTGTGGAC-3'. The PCR system was $20 \mu \mathrm{L}$ including $2 \mu \mathrm{L} 10 \mathrm{X}$ PCR buffer, $1.2 \mu \mathrm{L} 25 \mathrm{mM} \mathrm{MgCl}, 0.2 \mu \mathrm{M}$ dNTPs, $0.5 \mu \mathrm{M}$ primers, and $1 \mathrm{U}$ Taq enzyme (MBI). The reaction conditions were as follows: $95^{\circ} \mathrm{C}$ for $5 \mathrm{~min} ; 30$ cycles of $95^{\circ} \mathrm{C}$ for $1 \mathrm{~min}, 57^{\circ} \mathrm{C}$ (for the amplification of $\mathrm{GmPic}$ ) or $55^{\circ} \mathrm{C}$ (for the amplification of actin) for $1 \mathrm{~min}$, and $72^{\circ} \mathrm{C}$ for $1 \mathrm{~min}$; and $72^{\circ} \mathrm{C}$ extension for $5 \mathrm{~min}$. The experiment was repeated three times.

\section{Rapid amplification of cDNA end (RACE) generation of full-length GmPic}

Based on RT-PCR analysis results, the total RNA was extracted from soybean leaves with the long photoperiod treatment. The SMART ${ }^{\mathrm{TM}}$ RACE cDNA Kit (Clontech) was used for the amplification of the 5'- and 3'-ends of the GmPic cDNA according to manufacturer instructions. The primers that were used to amplify the 5'-end of the cDNA were 5'-TCTGGCATTTCTGGTGGTGGTGGTC-3' and 5'-TCCTGGGTCCCAATCCTTGCCATA A-3'. The primers that were used to amplify the 3'-end of the cDNA were 5'-ATGCCGTTTGCA AGTCGATCAAGC-3' and 5'-GGTTCTGTACACGTATGAGTTCTCTTC-3'. Two rounds of amplification were carried out. The PCR products were cloned and sequenced after separation on a $1 \%$ agarose gel.

\section{Sequence analysis}

The sequence was obtained after cloning, and vector sequences were removed. CAP3 
(http://bio.ifom-firc.it/assembly/assemble.html) was used for the splicing of the cDNA-AFLPand RACE-obtained gene sequences. The ORF Finder software was used for the analysis of gene structure, and BLASTX was used for the homology analysis. ClustalW (http://www.ebi. ac.uk/clustalw/) was used for the multiple sequence comparison.

\section{RESULTS}

\section{Flowering time difference of Zhong Dou 24 under different sunshine times}

Zhong Dou 24 seeds were sown on April 14, 2005. The first trifoliate grew on April 23, and the different sunshine treatments were applied after that. The field observation was conducted every 2 days, and the flowering dates were recorded. The field observations showed that under the short-time, natural sunshine land long-time, the first flower dates of Zhong Dou 24 were May 12, May 14, and June 13. The time periods were 24, 26, and 55 days from the seed emergence to the flowering. Compared with the natural light, the short-time sunshine treatment had little effect on the flowering time of Zhong Dou 24, but the long-time sunshine treatment greatly delayed the flowering time of Zhong Dou 24.

\section{Acquisition of GmPic differentially expressed fragments}

The flowering time of Zhong Dou 24 with the long-time sunshine treatment was later than that with the short-time and natural sunshine treatment, indicating that the longtime sunshine treatment may induce changes in gene expression that could lead to the delayed flowering time. The soybean leaf materials 5 days after the short-time, long-time, and natural sunshine treatment were analyzed by cDNA-AFLP. After amplification with the primer combination $\mathrm{E}(\mathrm{CG}) / \mathrm{M}(\mathrm{CT})$, the gene fragments with lengths of about $280 \mathrm{bp}$ were obtained and found to be up-regulated in the soybean leaves with long-time sunshine treatment (Figure 1). After the cloning, sequencing, and sequence comparison, the amino acid encoded by this gene was predicted to share about $80 \%$ homology with the mitochondrial phosphate carrier protein (Pic) in Drosophila, Aedes, and human; therefore, we named it GmPic (Figure 1).

\section{RT-PCR analysis of GmPic expression}

In order to verify that GmPic was the differentially expressed gene, RT-PCR analysis was used to analyze the GmPic expression in the leaves of Zhong Dou 24 with 5 days of short-time, long-time, and natural sunshine treatment. GmPic was only expressed in the soybean with the long-time sunshine treatment. The expression was undetectable in soybeans with the short-time sunshine and natural sunshine treatment (Figure 2). To further understand the expression pattern of GmPic, RT-PCR analysis was conducted to analyze the GmPic expression in the leaves of Zhong Dou 24 with 0-5 days of short-time, long-time, and natural sunshine treatment. GmPic was only expressed in leaves with 0-5 days of long-time sunshine treatment; GmPic expression was not detected in other samples (data not shown; Figure 2). 

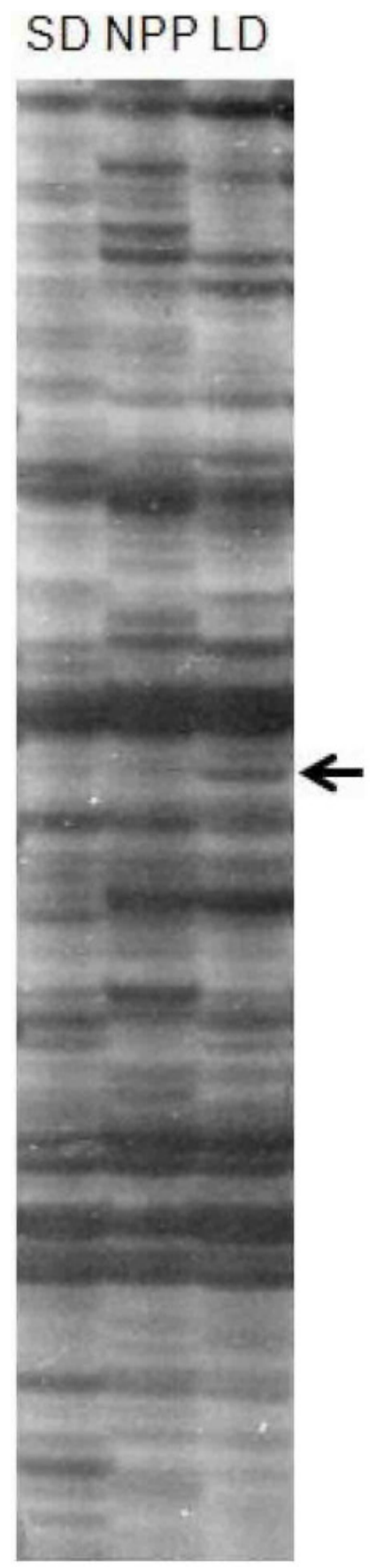

Figure 1. Gene expression of the leaves of soybean treated with different day length via the analysis of cDNA-AFLP. $\mathrm{SD}=$ short-day length; $\mathrm{NPP}=$ neutral photoperiod; $\mathrm{LD}=$ long-day length. The arrow indicates the differentially expressed GmPic. The primers were $\mathrm{E}(\mathrm{CG}) / \mathrm{M}(\mathrm{CT})$ used in cDNA-AFLP. 


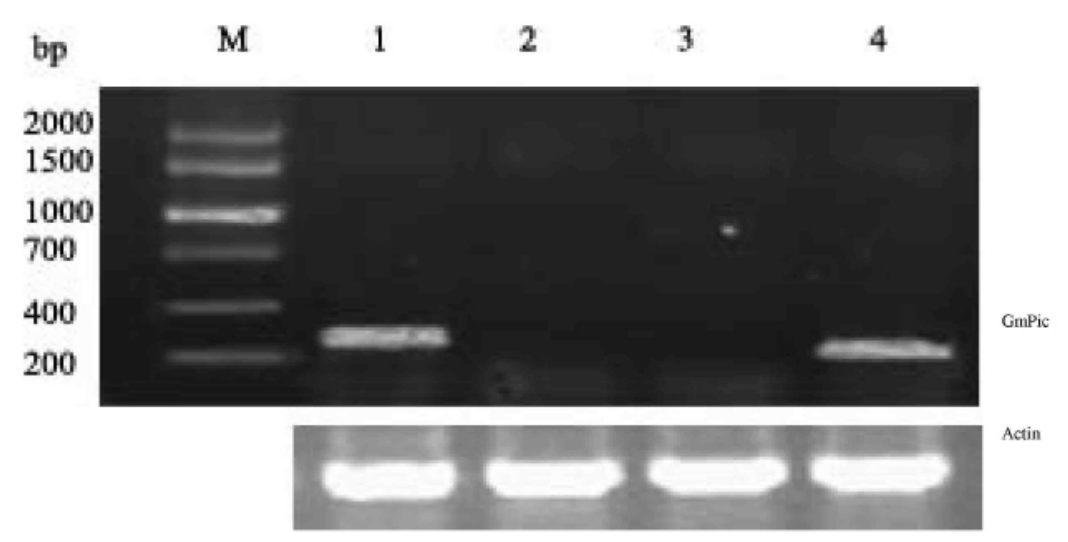

Figure 2. Expression of GmPic in leaves of Zhong Dou 24 treated with different day length by RT-PCR analysis. Lane $M=$ DNA marker $\delta$; lanes $1-4=$ samples of 0 and 5 days treated with SD, NPP, LP, respectively. For abbreviations, see legend to Figure 1.

\section{GmPic cDNA sequence obtained by RACE}

In order to further verify the function of GmPic, the 5'- and 3'-end sequences of GmPic were amplified by RACE. Through two rounds of amplification, approximately $400 \mathrm{bp}$ of the 5 '-end and $700 \mathrm{bp}$ of the 3'-end were amplified (Figure 3). The 5'- and 3'-ends of the amplified products were cloned and sequenced (Figure 3 ).

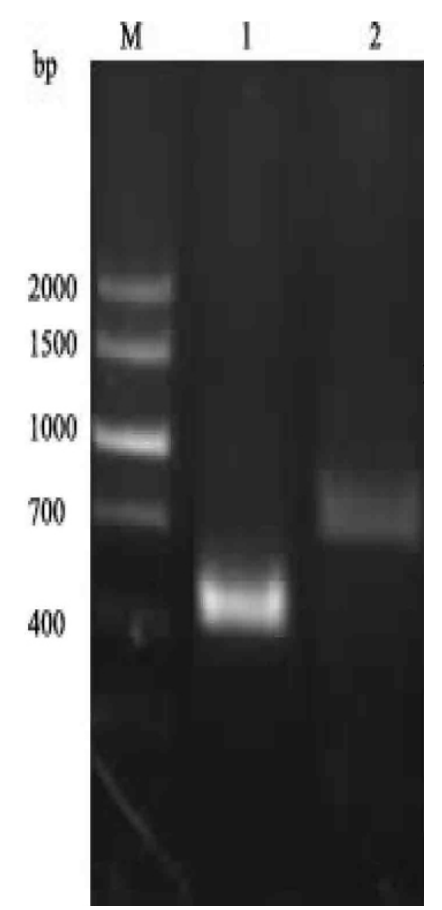

Figure 3. 5'- and 3'-RACE for GmPic. Lane $M=$ DNA marker $\delta$; lanes 1 and $2=5$ '- and 3'-RACE, respectively. 


\section{Sequence comparison and analysis}

The CAP3 software was used to determine the splice sites of the GmPic sequence that was acquired by cDNA-AFLP and RACE. The GmPic gene was found to have a length of 983 bp. Using the ORF Finder software, the sequence containing the complete open reading frame encoded 248 amino acids. Figure 4 shows the nucleotide sequence and the corresponding amino acid sequence of the gene.

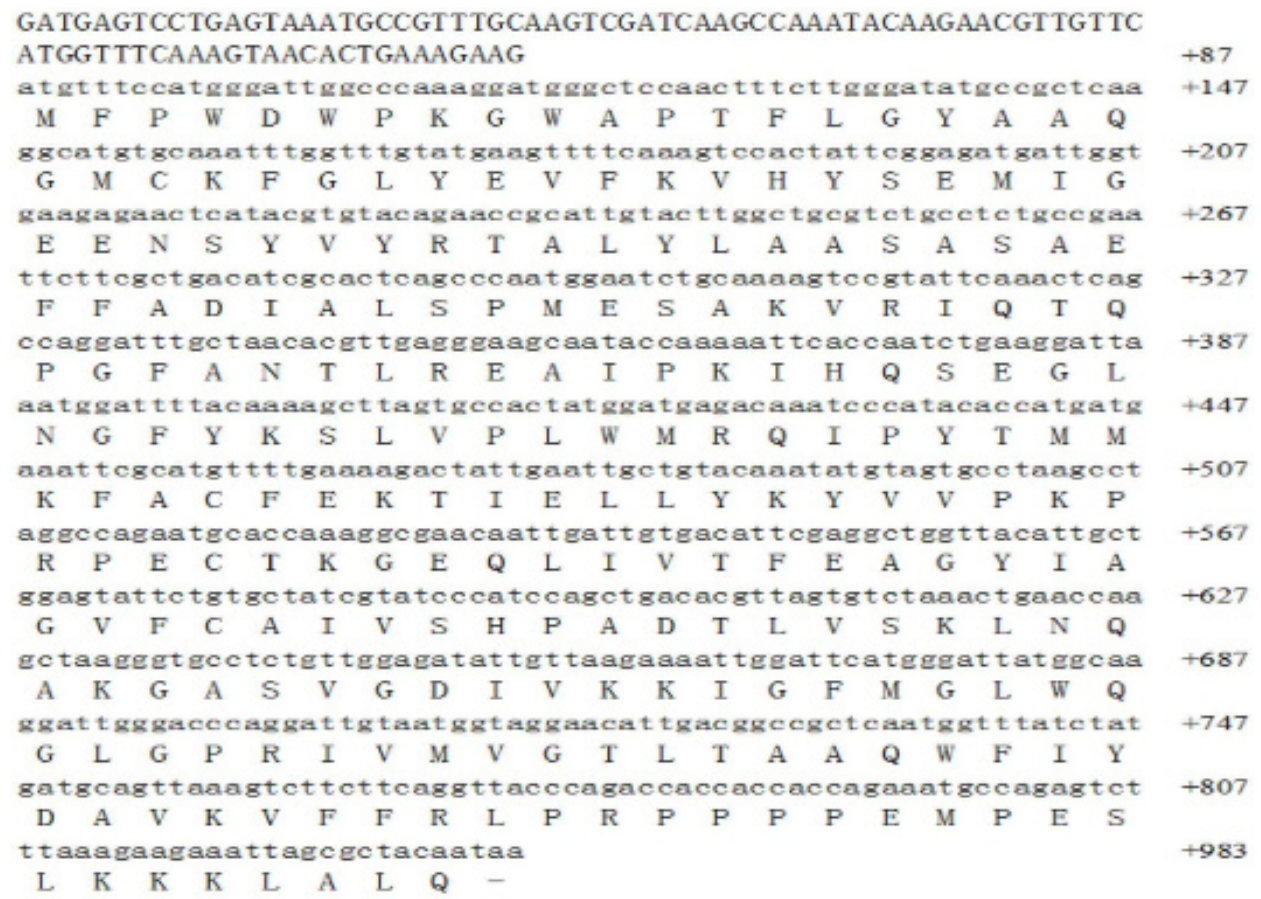

Figure 4. Nucleotide of GmPic and the deduced amino acids.

With the BLASTX software homologous sequence analysis, GmPic shared the highest homology with the amino acids of the mitochondrial phosphate transporter protein in Drosophila, Aedes, and human; the homology reached $80 \%$. It also shared $60 \%$ homology with amino acids encoded by the mitochondrial phosphate transporter genes in soybean, Lotus corniculatus, Medicago truncatula, maize, Arabidopsis, and rice. The sequence comparison results of GmPic and several plant homologs are shown in Figure 5.

The ClustalW software was used to determine the evolutionary relationship of the sequence homology. The GmPic gene was taken as one class; it shared the closest evolutionary relationship with the mitochondrial phosphate transporter gene of Arabidopsis and rice. The published mitochondrial phosphate transporter gene of the soybean was first clustered in the same class as the Lotus corniculatus mitochondrial phosphate transporter gene. It had a distant evolution relationship with GmPic (Figure 6). This indicated that the biological processes of the genes may differ from those of the known mitochondrial phosphate transporter gene. 


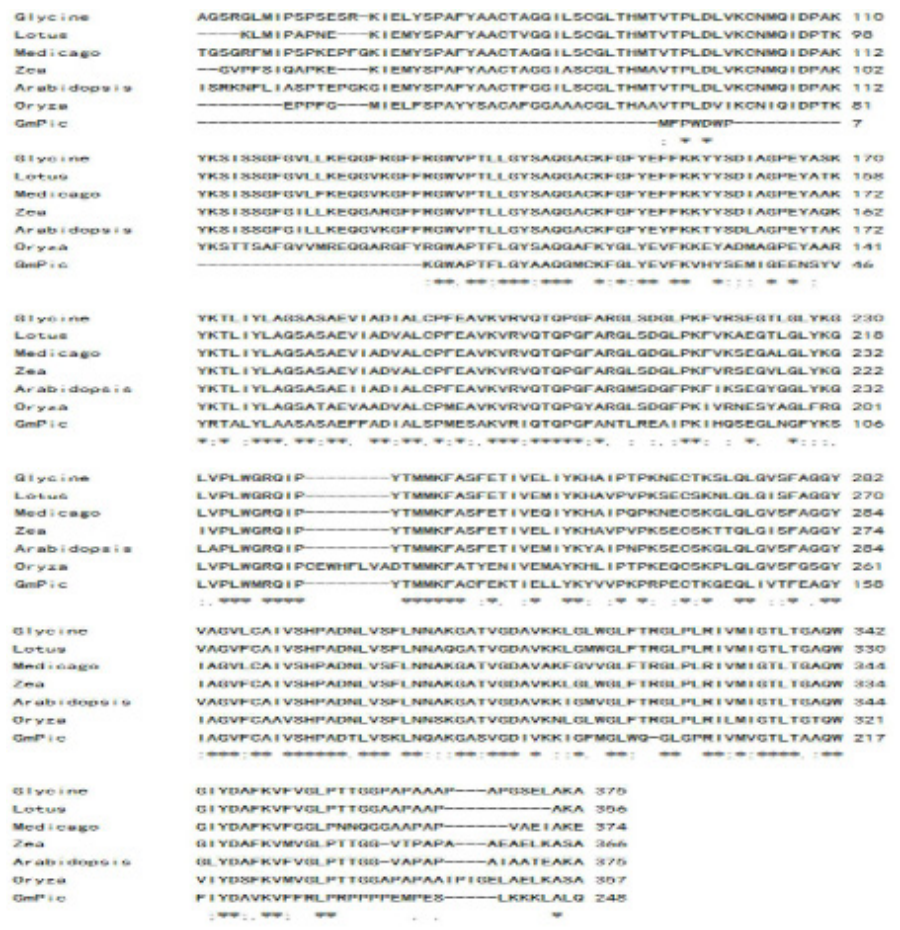

Figure 5. Comparison of predicted amino acid of mitochondrial phosphate transporter. [GenBank accession numbers (gi15241291: Arabidopsis, gi3318611: gi3318613: Glycine, Zea mays, gi18150857: Lotus japonicus, gi53828196: Medicago truncatula, gi51535220: Oryza)].

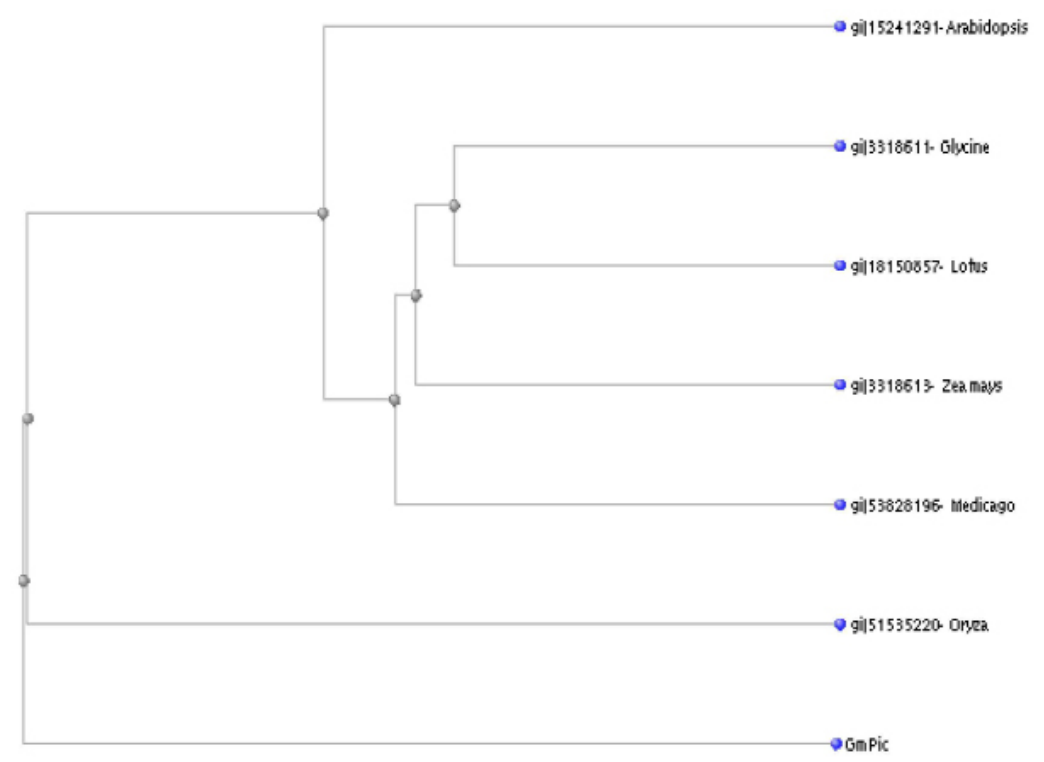

Figure 6. Phylogenetic tree of GmPic amino acids.

Genetics and Molecular Research 13 (2): 4380-4391 (2014) 


\section{DISCUSSION}

Through cDNA-AFLP analysis, the gene expression of the photoperiod-sensitive variety Zhong Dou 24 under 5 days of different sunshine treatment was analyzed. The results showed that GmPic was up-regulated in samples with long-time sunshine treatment. After RTPCR validation, GmPic was confirmed as the differentially expressed gene. Further analysis of GmPic dynamic expression in the development process from the pretreatment status to flowering showed that GmPic was up-regulated in samples with 0-5 days of long-time sunshine treatment. Combined with field observations that the flowering time of Zhong Dou 24 with the long-time sunshine treatment was much later than that with the short-time sunshine and natural sunshine treatment, our results indicated that GmPic may be a negative regulator that inhibits flowering. GmPic was expressed in the early stages of soybean growth and development, and the short-time sunshine would inhibit the gene expression and induce flowering, while the long-time sunshine would enhance its expression and thereby inhibit flowering.

In order to further analyze the GmPic function, the GmPic 3'-terminal sequence was obtained through RACE, but the 5'-terminal sequence was not obtained. The fragments that were obtained through cDNA-AFLP and RACE were spliced to obtain the 983-bp GmPic gene; it contained a complete reading frame encoding 248 amino acids. After the homologous sequence comparison, GmPic was found to share homology with the amino acid sequence of mitochondrial phosphate transporter protein in Drosophila, Aedes, and human; the homology reached $80 \%$. It also shared $60 \%$ homology with the mitochondrial phosphate transporter genes in soybean and Lotus corniculatus. Compared with the homologous gene, about 100 amino acids at the 5'-end were missing in the predicted GmPic amino acid sequence. It was presumed that the GmPic gene encoded a complete open reading frame. From the clustering view of evolutionary relationships, the GmPic gene was distant from the known mitochondrial phosphate transporter gene. It clustered in one group. Whether the role played by this gene is the same as that of the known gene requires further analysis.

There was no previous report about the isolation and function of the soybean mitochondrial phosphate transporter genes. However, the study results from rice and other crops indicated that this protein was a transmembrane protein located in the inner membrane of the mitochondrion. Its physiological function was to catalyze the transport of inorganic phosphate from the cytoplasm into the mitochondrion; it also provided the needed inorganic phosphate for ATP synthase to promote phosphorylation. The mechanisms in the regulation of Arabidopsis flowering suggested that the blue light receptors cry1 and cry2 were in the nonphosphorylated state in the dark and showed no activity. When exposed to the light, the phosphorylation would rapidly happen. Some studies showed that cry1 was able to receive light energy, excite electrons, and conduct the signal transmission only after it was phosphorylated. In the signal transmission process, time for coffee (TIC) acted as the switch for the blue-red light, and the input dose of the white light, which is the optical signal, would be controlled (Hall et al., 2003). TIC was an A functional domain (P-ring) that was able to bind ATP/GTP. Therefore, TIC was capable of binding to the protein kinase, which can be phosphorylated, and the rhythm was achieved through its phosphorylation (Ding et al., 2007). After the integration of the optical signals to the external environment and other signals, the signal enters the biological oscillator for processing into a variety of signals that the organism can identify. Through the mutual regulation of many genes, the organisms respond to these signals. Late 
elongated hypocotyl (LHY) and circadian and clock associated-1 (CCA1) were known as the key genes of biological oscillators (Alabadi et al., 2002). Some studies found that the two proteins can be phosphorylated by casein kinase II in plants; the phosphorylation and the formed composite body of CCA1 and DNA were very important for transcription (Mizoguchi et al., 2002; Pruneda-Paz and Kay, 2010). It was not difficult to find from the above findings that the phosphorylation played an important role in the sunshine time sensing and the regulation of the flowering process in Arabidopsis. However, its specific function and mechanism require further studies. The over-expression and inhibited expression of GmPic were studied in order to reveal its effects on soybean flowering.

\section{ACKNOWLEDGMENTS}

Research supported by the National Key Technologies R\&D Program of China (\#2011BAD35B06) and Research Fund for the Doctoral Program of Higher Education of China (\#20094601120001).

\section{REFERENCES}

Alabadi D, Yanovsky MJ, Mas P, Harmer SL, et al. (2002). Critical role for CCA1 and LHY in maintaining circadian rhythmicity in Arabidopsis. Curr. Biol. 12: 757-761.

Bachem CW, van der Hoeven RS, de Bruijn SM, Vreugdenhil D, et al. (1996). Visualization of differential gene expression using a novel method of RNA fingerprinting based on AFLP: analysis of gene expression during potato tuber development. Plant J. 9: 745-753.

Ding Z, Millar AJ, Davis AM and Davis SJ (2007). TIME FOR COFFEE encodes a nuclear regulator in the Arabidopsis thaliana circadian clock. Plant Cell 19: 1522-1536.

Gao PF (2008). A Soybean the Clock Genes GmLCL1/2 and GmTOC1 Cloning and Functional Characterization. Master's thesis, Qingdao Agricultural University, Qingdao.

Garner WW and Allard HA (1920). Effect of the relative length of day and night and other factors of the environment on growth and reproduction in plants. J. Agric. Res. 18: 553-606.

Hall A, Bastow RM, Davis SJ, Hanano S, et al. (2003). The TIME FOR COFFEE gene maintains the amplitude and timing of Arabidopsis circadian clocks. Plant Cell 15: 2719-2729.

Hayama R and Coupland G (2004). The molecular basis of diversity in the photoperiodic flowering responses of Arabidopsis and rice. Plant Physiol. 135: 677-684.

Hayama R, Izawa T and Shimamoto K (2002). Isolation of rice genes possibly involved in the photoperiodic control of flowering by a fluorescent differential display method. Plant Cell Physiol. 43: 494-504.

Hayama R, Yokoi S, Tamaki S, Yano M, et al. (2003). Adaptation of photoperiodic control pathways produces short-day flowering in rice. Nature 422: 719-722.

Hecht V, Foucher F, Ferrandiz C, Macknight R, et al. (2005). Conservation of Arabidopsis flowering genes in model legumes. Plant Physiol. 137: 1420-1434.

Ke HY(2006). Cloning and Function Analysis of a Homologous Gene ZEITLUPE Involed in Circadian Clock in Soybean. Master's thesis, Fujian Agriculture and Forest University, Nanping.

Kojima S, Takahashi Y, Kobayashi Y, Monna L, et al. (2002). Hd3a, a rice ortholog of the Arabidopsis FT gene, promotes transition to flowering downstream of Hd1 under short-day conditions. Plant Cell Physiol. 43: 1096-1105.

Liu B, Kanazawa A, Matsumura H, Takahashi R, et al. (2008). Genetic redundancy in soybean photoresponses associated with duplication of the phytochrome A gene. Genetics 180: 995-1007.

Liu H, Wang H, Gao P, Xu J, et al. (2009). Analysis of clock gene homologs using unifoliolates as target organs in soybean (Glycine max). J. Plant Physiol. 166: 278-289.

Mizoguchi T, Wheatley K, Hanzawa Y, Wright L, et al. (2002). LHY and CCA1 are partially redundant genes required to maintain circadian rhythms in Arabidopsis. Dev. Cell 2: 629-641.

Pittendrigh CS and Minis DH (1964). The entrainment of circadian oscillations by light and their role as photoperiodic clocks. Am. Nat. 108: 261-295. 
Pruneda-Paz JL and Kay SA (2010). An expanding universe of circadian networks in higher plants. Trends Plant Sci. 15: 259-265.

Sha AH, Cai SP, Zhang XJ, Wu XJ, et al. (2006). Cloning and expression of GmCO and GmFT controlling the flowering time of soybean. Xi Bei Zhi Wu Xue Bao 26: 1996-2000.

Vos P, Hogers R, Bleeker M, Reijans M, et al. (1995). AFLP: a new technique for DNA fingerprinting. Nucleic Acids Res. 23: 4407-4414.

Yang ZP, Zhang XJ, Cai SP, Qiu DZ, et al. (2001a). Response of short juvenility soybean varieties to photoperiods. II. Response to long daylength. Soybean Sci. 20: 191-196.

Yang ZP, Zhang XJ, Cai SP, Qiu DZ, et al. (2001b). Response of short juvenility soybean varieties to photoperiods. II. Response to short daylength. Chin. J. Oil Crops Sci. 23: 35-39.

Yanovsky MJ and Kay SA (2003). Living by the calendar: how plants know when to flower. Nat. Rev. Mol. Cell Biol. 4: 265-275.

Zhao L, Luo QL, Han YP and Li WB (2007). Soybeans in the dark under inducing conditions differentially expressed cDNA library construction and analysis. Da Dou Ke Xue 26: 134-139. 